\title{
PGP2X: Principal Geometric Primitives Parameters Extraction
}

\author{
Zahra Toony, Denis Laurendeau, and Christian Gagné \\ Computer Vision and System Laboratory, Department of Electrical and Computer Engineering, Université Laval, Québec, \\ $Q C$, Canada \\ zahra.toony.1@ulaval.ca,denis.laurendeau@gel.ulaval.ca,christian.gagne@gel.ulaval.ca
}

Keywords: $\quad$ Parameter extraction, Geometric primitives, Principal Component analysis.

\begin{abstract}
In reverse engineering, it is important to extract the $3 \mathrm{D}$ geometric primitives that compose an object. It is also important to find the values of the parameters describing each primitive. This paper presents an approach for the estimation of the parameters of geometric primitives once their type is known using 3D information. The primitives of interest are planes, spheres, cylinders, cones, tori and partial instances of the latter four types. The proposed approach extends methods found in the literature for planes, spheres, cylinders and cones and proposes a new method for dealing with tori. The results of the proposed method are compared to approaches found in the literature as well as with ground truth values. The proposed method can be applied to the estimation of parameters of geometric primitives of synthetic CAD models as well as for models of real objects acquired with 3D scanners.
\end{abstract}

\section{INTRODUCTION}

Recognizing 2D objects and finding their primitives was one of the most popular topics in computer vision and still remains a challenging task. Accurate 3D scanners have started a challenging domain of research in object detection and recognition which consists of recognizing objects based on their geometry rather than their appearance. Such 3D sensors capture the geometry of the objects and allow the type of $3 \mathrm{D}$ objects to be recognized and their descriptive parameters to be estimated.

Estimating the parameters of 3D models can be helpful in different applications such as reverse engineering, 3D model retrieval, and classification of 3D models. Some methods have been proposed to recognize different types of primitives [Toony et al., 2014, Osada et al., 2002, Kazhdan et al., 2003, Zhu et al., 2012]. Once a primitive has been recognized, we need an approach to extract the parameters of each primitive. Some methods, [Attene et al., 2006, Attene and Patanè, 2010, Fayolle and Pasko, 2013], first fit a primitive model to the data and then find the parameters of the model. Our goal is to extract the primitive parameters directly from the original model without a fitting process. The motivation of our work is to estimate the parameters of the principal types of primitives encountered in reverse engineering applications and object modelling. Since $95 \%$ of industrial objects can be described by spheres, planes, cones, cylinders, and tori [Rabbani and Van Den Heuvel, 2005], we propose an approach for estimating the descriptive parameters of these types of primitives.

The rest of the paper is organized as follows. Related work is presented in Section 2. The proposed approach for extracting the parameters of each primitive type is explained in detail in Section 3. Section 4 presents experimental results and demonstrates the efficiency of our approach in different situations and in comparison with other methods. Section 4 also presents results obtained on real scans of 3D objects composed of the primitives of interest. Finally, we conclude the paper in Section 5.

\section{RELATED WORK}

In order to estimate the parameters of primitives, different approaches can be considered. The methods that have been proposed so far can be divided into two categories. The first category consists of techniques that segment the model at first and then determine the type of each segment as well as their descriptive parameters. Authors usually use different primitives in order to identify the type of each segment. The second category contains methods which start directly from the original model without any pre-processing such as segmentation. These methods apply a fitting process to identify the type of primitive and then the pa- 
rameters of the best fitted primitive are returned. Our approach deals with original models without any preprocessing but the assumption is made that the model contains only one primitive which can be in the first category but without a prior segmentation process. Since several methods have been proposed to determine the type of primitives, [Toony et al., 2014, Osada et al., 2002, Kazhdan et al., 2003, Zhu et al., 2012], in this paper, we assume that the type of each primitive is known and focus on an accurate estimation of primitive parameters.

The methods in the first category require a segmentation step and a non-linear fitting approach with reliable initial seeds. One of the methods in this category, presented in [Lukács et al., 1998], uses a segmentation approach based on initial seeds and region growing. The segmentation and fitting stop based on the fitting error. This method works for spheres, cylinders, cones, and tori. The method is sensitive to the choice of the initial seed and is also very time consuming.

Another approach in this category is presented in [Benko et al., 2002]. The authors assume that the input point cloud is already segmented into primitives and the type of primitive is already known. They suppose that there is a set of parametrized objects for which the parameters need to be estimated. They consider some "primary objects" such as surfaces, curves, etc. and some "auxiliary objects" that describe the constraints between primary objects. A fitting process is used in order to find the parameters of the primary and auxiliary objects.

In the paper presented in [Fayolle and Pasko, 2013], which belongs the first category, the authors use a set of primitives from a user-specified list of primitives. They then fit these primitives and extract the points that correspond to the best fitted primitive and the parameters are obtained from the best fit. The list of primitives includes spheres, cylinders, planes, tori, cones, and super-ellipsoids.

The approaches in the second category extract the primitives directly from the input point cloud using RANSAC-based methods for instance [Li et al., 2011, Schnabel et al., 2007, Bolles and Fischler, 1981, Fischler and Bolles, 1981]. In [Schnabel et al., 2007], an automatic method is presented based on random sampling which detects planes, spheres, cylinders, cones, and tori. This RANSAC-based method is time consuming like all random based methods and it also depends on the selected points. The method presented in [Olson, 2001] is splitting and pruning the parametric space in order to implement a faster algorithm. Some other methods use the Gaussian sphere for extracting primitives [Chaperon et al., 2001, Rabbani and Van
Den Heuvel, 2005, Liu et al., 2013].

The methods presented in [Borrmann et al., 2011, Kotthäuser and Mertsching, 2012] are extracting planes only but other methods are proposed to extract cylinders [Bolles and Fischler, 1981, Lozano-Perez et al., 1987, Chaperon et al., 2001, Rabbani and Van Den Heuvel, 2005, Liu et al., 2013].

A hierarchical fitting approach which deals directly with input data is introduced in [Attene et al., 2006] and [Attene and Patanè, 2010]. The method presented in [Attene et al., 2006] produces a binary tree of primitives, extracting planes, spheres, and cylinders. Another approach [Attene and Patanè, 2010] exploits more accurate hierarchical clustering in order to extract more primitives such as planes, spheres, cylinders, cones, and tori. Since we compare our results with these approaches, they are described in detail in the following section.

\subsection{A Review of Two Hierarchical Fitting Approaches}

\subsubsection{Method proposed in [Attene et al., 2006]}

The basic idea of the method presented in [Attene et al., 2006] is to merge neighboring triangles into representative clusters. The idea is to build a dual graph from the input mesh model. At the beginning of the algorithm, each node of the graph represents a cluster. The authors assign a weight to each edge of the graph. They then build a priority queue based on the edges' weights and they merge the edges with the minimum weights in the priority queue. After each merging operation, edge weights and clusters are updated. The algorithm stops based on some criteria. Fitting is applied to each cluster and the primitive which has the minimum evaluation error is selected.

Planes, spheres, and cylinders are considered. To extract planes, the authors use a classical method based on PCA (Principal Component Analysis) [Garland et al., 2001, Cohen-Steiner et al., 2004]. First the covariance matrix of each cluster is computed. The normal of the plane is the eigenvector corresponding to the smallest eigenvalue of the covariance matrix. The fitting error is calculated afterwards.

In order to find the parameters of the spheres (radius and location of the center), several different radii and centers are tested and those that minimize the distance between the points and the fitted sphere are selected. These parameters can be found by solving a Gauss-Newton minimization problem [Scales, 1985] but, as mentioned in the paper [Attene et al., 2006], this is a time consuming process so an algebraic distance approach [Pratt, 1987] has rather been used to 


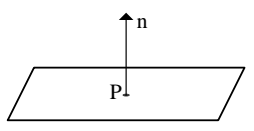

(a)

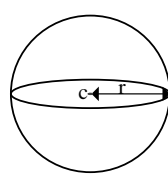

(b)

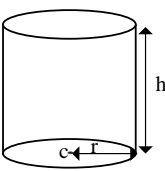

(c)

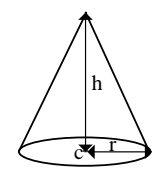

(d)

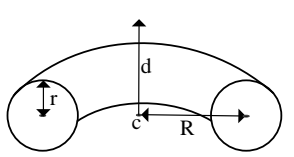

(e)

Figure 1: The parameters extracted for: (a) plane, (b) sphere, (c) cylinder, (d) cone, and (e) torus.

determine the sphere's parameters.

For cylinders, the parameters that need to be estimated are the radius, the axis direction, and a point belonging to the axis which is called the center. In order to estimate the axis direction, they first compute a covariance matrix on the edges of the dual graph. Then, they select the eigenvector related to the largest eigenvalue of the covariance matrix as the axis of the cylinder. A plane is then defined with a normal in the same direction of the axis of the cylinder and a point as the center of mass of the cylinder points. Then, all points of the cylinder are projected onto the plane and, using a direct circle fitting approach, the radius and the center location of the created circle on the plane are computed. Finally the fitting error is calculated.

\subsubsection{Method proposed in [Attene and Patanè, 2010]}

In this method, the authors consider spheres, planes, cylinders, cones, and tori. They present an algorithm to convert a 3D surface into a hierarchical representation. A k-nearest neighbor graph is built on the input point-set which is a time consuming process. The procedure for estimating the parameters of planes and spheres is the same as the one presented in [Attene et al., 2006]. For cylinders, the Gaussian sphere concept is used. First the normals of the cylinders are mapped on the Gaussian sphere, the axis of the cylinder is found using PCA. A plane is then mapped on the points located on the Gaussian sphere and, finally, all of these points are projected onto the plane and a direct circle fitting approach is used to find the radius of the projected circle.

For cones, the normals are mapped on the Gaussian sphere and a plane is fitted on the normals. PCA is used to find the normal vector to the plane. The apex of the cone is found using a minimization process and, finally, the semi-apical angle that shows the deviation of the cone surface from the axis is computed.

For tori, a center point, a height vector and a radius are calculated. The curvature properties of the torus are used to estimate the height vector and the small radius of the torus. Each point of the torus is moved on the opposite direction of its normal with a signed distance obtained from the curvature value. So, all of the points are placed on a circular axis. The plane passing through the transformed points is then found. The direction of the plane's normal is the height vector. These transformed points are projected onto the plane, and the center and the radius are found by applying a circle fitting algorithm on the projected points.

In this paper, we compare our results with [Attene and Patanè, 2010]'s method and also with the result of a non-linear least-squares fitting algorithm of the LSGE library developed for fitting primitives [LSGE, 2004]. In our method, we extract the parameters of planes, spheres, cylinders, cones, and tori as well as partial instances of the former four types of primitives. The methodology for extracting the parameter values of each type of primitive is detailed in the following section.

\section{PROPOSED METHOD (PGP2X)}

Regardless of the type of input data, mesh or point cloud, we estimate the normal at each vertex of each primitive. If the model is a mesh, we consider the normal at a vertex as the average between the normals of its connected faces. The normal to a triangular face is the cross product of its two adjacent sides. If the model is a point cloud, we compute the normal at each point using the method presented in [Zhang et al., 2013] which is a robust normal estimation method based on a low-rank subspace clustering approach. A covariance analysis of the neighborhood is used to find the smooth and sharp regions around the points. A guiding matrix using an unsupervised learning process based on neighborhood features is built. Finally, the anisotropic neighborhoods are segmented into some isotropic neighborhoods using the guiding matrix and the low-rank subspace clustering approach. For a point near smooth regions, the normal can be obtained easily and accurately but for points near sharp regions, the normal is estimated as the normal of a fitted plane to the consistent sub-neighborhoods [Zhang et al., 2013].

Now that the procedure for obtaining the normals 


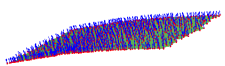

(a)

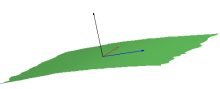

(b)

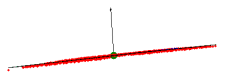

(c)
Figure 2: (a) Real scanned plane with vertices and normals, (b) principal components obtained from vertices, (c) fitted plane using the normal obtained from PCA and the median of vertices as the point.

to the vertices has been presented and the type of each primitive is known, we go through the details of parameter extraction for each primitive. The primitives that are considered in this paper are planes, spheres, cylinders, cones, and tori as well as partial models of the latter four types of primitives. Based on the method presented in [Toony et al., 2014], the type of primitive is found. The parameters are extracted for each primitive type are listed in figure 1 .

\subsection{Plane}

The parameters of a plane are the normal to the plane, $\vec{n}$, as well as a point $p$ lying on the plane (figure 1 (a)). In order to find the normal to the plane, a good method is the Principal Component Analysis (PCA). We apply the PCA on the vertices of the input data. The eigenvector corresponding to the smallest eigenvalue is selected as the normal to the plane. The median of the points is chosen as a point lying on the plane.

Figure 2 presents a scanned plane. Part (a) shows the plane with vertices and normals; the principal components are presented in part (b) and, finally, the fitted plane using the normal extracted from PCA and the median of the points are shown in part (c).

\subsection{Sphere}

The parameters that need to be extracted for a sphere are the center $c$ and the radius $r$ (see figure 1 (b)). In order to estimate the two parameters, different methods can be used. The simplest but the most time consuming is the fitting approach. In this paper we rather use a fast method which relies on four non-coplanar points [Schmitt, 2005]. A sphere can be extracted uniquely from four points if they are not on the same plane [Schmitt, 2005]. The details of the approach are presented in the following:

1. In order to have four non-coplanar points, we select three non-collinear points $P_{1}, P_{2}$, and $P_{3}$. Then we find the plane passing through these three points and the fourth point is the one whose dot product with the plane's normal is nonzero, meaning that this fourth point is non-coplanar with the other three points.
2. Once four points have been selected, we need to solve the following determinant equation:

$$
\left|\begin{array}{ccccc}
x^{2}+y^{2}+z^{2} & x & y & z & 1 \\
x_{1}^{2}+y_{1}^{2}+z_{1}^{2} & x_{1} & y_{1} & z_{1} & 1 \\
x_{2}^{2}+y_{2}^{2}+z_{2}^{2} & x_{2} & y_{2} & z_{2} & 1 \\
x_{3}^{2}+y_{3}^{2}+z_{3}^{2} & x_{3} & y_{3} & z_{3} & 1 \\
x_{4}^{2}+y_{4}^{2}+z_{4}^{2} & x_{4} & y_{4} & z_{4} & 1
\end{array}\right|=0 .
$$

3. This determinant can be written as:

$$
\left(x^{2}+y^{2}+z^{2}\right) M_{11}-x M_{12}+y M_{13}-z M_{14}+M_{15}=0 .
$$

4. Considering $x^{2}+y^{2}+z^{2}=r^{2}$, we write the sphere equation as follows:

$$
\left(x-x_{0}\right)^{2}+\left(y-y_{0}\right)^{2}+\left(z-z_{0}\right)^{2}-r_{0}^{2}=0 .
$$

5. After equating the expansion of equation 3 with equation 2 , the parameters of the sphere are obtained as,

$$
\begin{aligned}
& x_{0}=+0.5 \frac{M_{12}}{M_{11}}, y_{0}=-0.5 \frac{M_{13}}{M_{11}}, z_{0}=+0.5 \frac{M_{14}}{M_{11}}, \\
& r_{0}=x_{0}^{2}+y_{0}^{2}+z_{0}^{2}-\frac{M_{15}}{M_{11}} .
\end{aligned}
$$

see [Schmitt, 2005] for the definition of each $M_{1 i}$.

\subsection{Cylinder}

For a cylinder, we need to extract the direction of the axis, $\vec{d}$, the height of the cylinder, $h$, the center, $c$, and the radius, $r$, of the basis as presented in figure 1 (c). The proposed method to estimate the parameters of cylinders is presented in the following steps:

1. Map all normals of the cylinder on the Gaussian sphere. This creates a great circle on the sphere.

2. Find the plane passing through the normals. To achieve this, we apply PCA on the normals and we select the vector corresponding to the smallest eigenvalue as the normal to the plane which is the direction of the cylinder's axis, $\vec{d}$. Selecting one of the points on the Gaussian sphere and the normal direction of the plane, we have the plane that is passing through the normals on the Gaussian sphere.

3. Once the plane has been determined, all points of the cylinder are projected onto the plane which creates a circle on the plane.

4. In order to obtain the radius of the circle, most methods, such as the one proposed by Attene in [Attene et al., 2006] and [Attene and Patanè, 


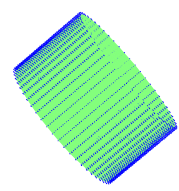

(a)

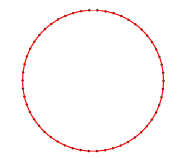

(d)

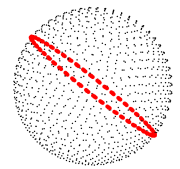

(b)

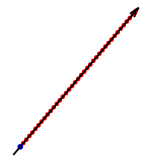

(e)

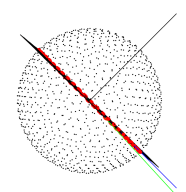

(c)

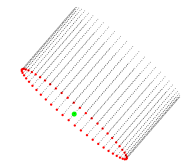

(f)

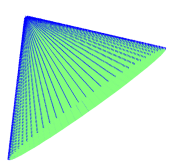

(a)

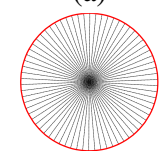

(d)

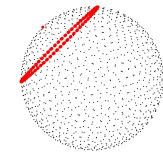

(b)

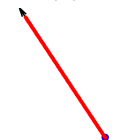

(e)

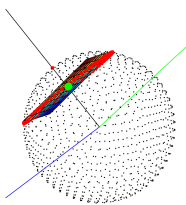

(c)

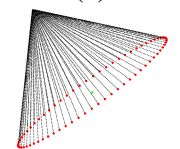

(f)
Figure 3: Extracting the parameters of a cylinder: (a) 3D model of a cylinder with its normals; (b) normals of the cylinder on the Gaussian sphere; (c) the fitted plane on the normals; (d) projected points on the plane and the result of Taubin's circle fitting; (e) projected cylinder points on the plane's normal (axis vector), the blue point shows the minimum value; and (f) the basis of the cylinder with it's center (green dot).

2010], use direct circle fitting. In this paper, we rather use Taubin's method [Taubin, 1991] to estimate the radius. Taubin's method is fast and robust and it works well even in the case of a partial circle with a small arc [Chernov, 2009]. Taubin's method is more stable than the circle fitting approach proposed by Kasa [Kasa, 1976] and is faster than the direct circle fitting by Pratt [Pratt, 1987].

5. To estimate the center of the cylinder, if the $2 \mathrm{D}$ center of the Taubin's fitted circle is transformed into $3 \mathrm{D}$, it returns a point on the axis vector, as in Attene's method, but is not exactly the center of the cylinder base. So, we first determine the points right at the base of the cylinder and then we extract the center. To achieve this, all points on the cylinder are projected onto the axis vector and the minimum projected value is found. Afterwards, all cylinder points whose projected values are in a small neighborhood of the minimum value are selected. This creates a circle as shown in figure 3 . Then, three points on this circle are chosen, knowing the radius $r_{0}$ from the previous step, the general circle equation is solved, $\left(x-x_{0}\right)^{2}+\left(y-y_{0}\right)^{2}+\left(z-z_{0}\right)^{2}=r_{0}^{2}$, to find the center, $c=\left(x_{0}, y_{0}, z_{0}\right)$,of the cylinder base.

6. In the last step, to find the height of the cylinder, the difference between the min and the max values of the projected points on the axis vector are found and the height is computed as $h=\max -\min$.
Figure 4: Extracting the parameters of a cone: (a) 3D model of a cone with its normals; (b) normals of the cone on the Gaussian sphere; (c) the fitted plane on the normals; (d) projected points on the plane with Taubin's circle fitting; (e) projected cone points on the plane normal (axis vector), the blue point shows the minimum value; and (f) the base of the cone with its center (green dot).

\subsection{Cone}

The parameters of a cone that need to be extracted are the axis direction, $\vec{d}$, the height of the cone, the radius, $r$, and the center of the cone base, $c$. In the following we explain the details of our approach to extract these parameters:

1. The first step is similar to the case of cylinders and consists in mapping all normals of the cone on the Gaussian sphere. This creates a small circle on the sphere (figure 4 (b)).

2. A plane is fitted through the normals. The direction of the plane's normal is the direction of the axis vector.

3. All of the 3D points of the cone are projected onto the plane which creates several circles. Using Taubin's method, circles are fitted and the one with the greatest radius is selected.

4. To estimate the height of the cone, all 3D points are projected onto the axis vector, and the difference between the minimum and the maximum projected values is considered as the height of the cone.

5. In order to estimate the center of the cone base, the base of the cone must be estimated first, as for cylinders. In a case of an upward cone, all $3 \mathrm{D}$ points corresponding to the minimum projected value on the axis direction, or the points related to the maximum projected value, in the case of a downward cone, are located on the cone base. For the estimation to be independent from the direction of the cone, the points in a small neighborhood of the minimum and the maximum projected values on the axis direction are selected. So, two 
sets are considered, one around the minimum projected value and the other one includes the points around the maximum projected value. The set that consists of one point, the apex, is rejected. As for cylinders, knowing the radius and three points of the base, the circle equation is solved in order to determine the center of the cone base.

This procedure is applied on a cone that is presented in figure 4.

\subsection{Torus}

Estimating the parameters of the torus primitive is the most challenging task of primitive parameter extraction. In fact, approaches reported in the literature following the research presented by [Attene and Patanè, 2010] have failed. However, here, we present a novel algorithm to extract the parameters of a torus. The method is accurate for different instances of tori. The parameters to be extracted are the direction vector, $\vec{d}$, which is orthogonal to the torus model, the center, $c$, the radius for the great circle, $R$, and the radius for the small circle, $r$. In the following we explain the details of the approach that is proposed to extract these parameters:

1. PCA is applied on the 3D points of the torus model. The eigenvector corresponding to the smallest eigenvalue is considered as the direction vector, $\vec{d}$.

2. A plane is located with $\vec{d}$ as its normal vector and the point that is the average of the points on the torus. This point is considered as the center, $c$, of the torus.

3. All 3D points of the torus are projected onto

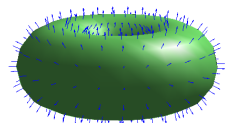

(a)

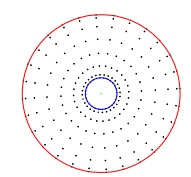

(c)

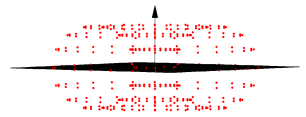

(b)

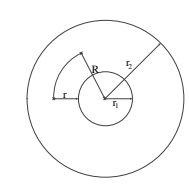

(d)
Figure 5: Extracting the parameters of a torus: (a) 3D model of a torus with its normals; (b) 3D points of the torus with the PCA fitted plane and the direction vector; (c) projected points on the plane with the greatest and smallest fitted circles, red and blue circles respectively; and (d) two new radii, $r_{1}$ and $r_{2}$ are used to compute $R$ and $r$. the plane. This produces several circles. Using Taubin's method, the parameters of these circles are found. The smallest and the greatest radii, $r_{1}$ and $r_{2}$ respectively, are used to compute the radii of the torus model. As presented in figure 5 (d), the two radii, $r_{1}$ and $r_{2}$ are used to compute $R$ and $r$, with the following equations:

$$
\begin{gathered}
R=r_{1}+\frac{r_{2}-r_{1}}{2}=\frac{r_{1}+r_{2}}{2}, \\
r=\frac{r_{2}-r_{1}}{2} .
\end{gathered}
$$

This methodology works well for complete CAD models or real scans of complete tori. However, for partial tori, especially small ones, the eigenvector corresponding to the smallest eigenvalue is not always the vector perpendicular to the model. To deal with such cases, the three principal components of the points on the models are computed. The vector associated with each component is considered in turn as the normal to the torus plane which produces three different projection results. Figure 6 depicts these different projections.

In order to identify the right plane, for each of these three conditions, the points outside of a margin distance $\varepsilon$ to the plane are ignored and then the distance of the remaining points to the plane are calculated. When the diagram of the sorted distances for each plane is plotted, the three diagrams in figure 7 plot (a)-(c) are obtained. For a torus model, especially CAD models, the points on the torus are located on circles but on different levels. We thus have several points at the same distance to the plane and then

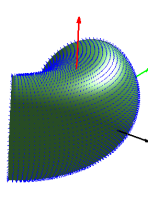

(a)

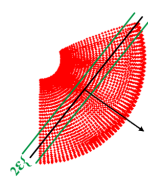

(b)

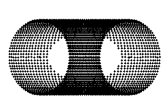

(e)

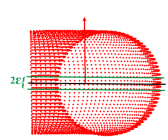

(c)

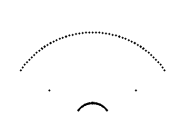

(f)

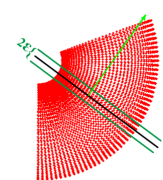

(d)

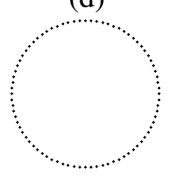

(g)
Figure 6: Finding the right direction of the plane for a partial torus: (a) the 3D CAD model of a partial torus with its three principal components; (b) selecting the eigenvector corresponding to the smallest eigenvalue as the normal to the plane; (c) selecting the eigenvector corresponding to the second smallest eigenvalue as the normal to the plane; (d) selecting the eigenvector corresponding to the largest eigenvalue as the normal to the plane; and (e)-(g) projecting $3 \mathrm{D}$ points on the plane presented in (b), (c), and (d) respectively. 


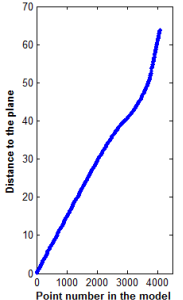

(a)

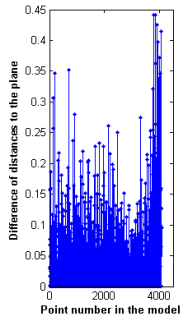

(d) $S F M=0.0236$

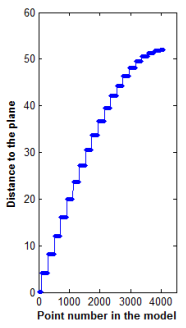

(b)

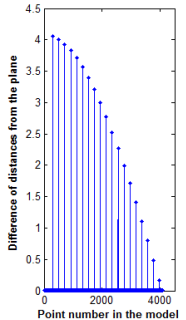

(e) $S F M=0.0001$

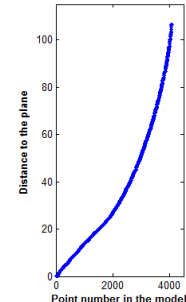

(c)

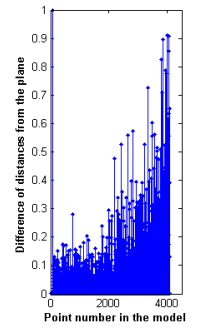

(f) $S F M=0.0171$
Figure 7: Diagram of the distances to the plane: (a)-(c) diagrams of the distances to the planes presented in figure 6 (b)-(d), respectively; while (d)-(f) show difference of distances between contiguous points of the diagrams presented in plot (a)-(c), respectively. The spectral flatness value is calculated for the difference of distances diagrams, plot (d)(f) and the values are presented for each diagram. The minimum value ((e) in this case) corresponds to the right direction of the plane.

another series of points with a different common distance to the plane and so on for the different levels. The difference of sorted distances shows many zero values in the diagram with sharp steps which is caused by the points with the same distance to the plane. If the plane is the right one, with the normal perpendicular to the model, the diagram of differences of distances has several zeros and sharp peaks. However if the plane is the wrong one, there is no regularity between the distance of points from the plane. The flatness of the diagram of differences of distances can be considered as a measure that can be used to find the right plane. This regularity is more observable when the model is a CAD model as it is the case for figure 7. However, the approach has also been applied successfully to real scanned models.

When the plane is the wrong one, there is no regularity in the distribution of the distances of the points to the plane. The Kurtosis of the difference of distances diagram or its flatness are parameters that could be used to find the most regular one. Based on our experiments, flatness has demonstrated to be more efficient than Kurtosis. For this reason, the Spectral Flatness Measure (SFM) [Johnston, 1988] was used to estimate the flatness. SFM is obtained by com-

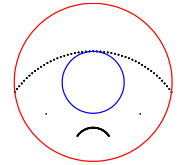

(a)

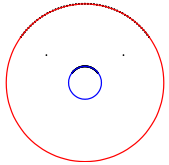

(b)
Figure 8: Finding the parameters of the circles for the partial torus of figure 6 using (a) Taubin's method and (b) the RCD approach. The results show that Taubin's method is not suitable for two partial circles while the RCD approach is able to extract the parameters correctly.

puting the ratio between the geometric mean of the diagram and the arithmetic mean of the diagram:

$$
S F M=\frac{\sqrt[N]{\prod_{i=1}^{N} y_{i}}}{\frac{\sum_{i=1}^{N} y_{i}}{N}}
$$

where, $N+1$ is the number of points, $y_{i}$ is the difference of distance to the plane between point $i$ and $i+1$.

Using the spectral flatness, the direction vector of the torus model, $\vec{d}$, can be found for both complete and partial models. Once the plane is obtained, the points within a small margin distance $\varepsilon$ to the plane are selected and are projected onto the plane. For a complete torus, this creates two full circles and Taubin's method can be used to find the radius of the two circles and, using equation 5 and 6 , the two radii of the torus can be computed. For partial models only two partial circles are observed and Taubin's method, which also works in the case of a single partial circle, fails to find the correct radii since there are two partial circles. The Randomized Circle Detection (RCD) method can be used instead [Chen and Chung, 2001]. Figure 8 shows that the Taubin's method fails for the partial torus of figure 6 but works well for a complete torus as observed in figure 5 plot (c).

RCD can also be applied to full circles but since Taubin's method is fast and accurate, RCD is only used in the case of partial tori. In order to decide which approach to be used, Taubin's method is applied first and then the small and great circles are found. The points located on these two circles are removed. If more than $30 \%$ of the points remain, RCD is used instead.

The Randomized Circle Detection [Chen and Chung, 2001] was introduced for detecting circles on images. Here, the RCD is used to detect circles made of $2 \mathrm{D}$ points, which are $3 \mathrm{D}$ points projected on a plane. RCD begins with a set of pixels $V$, and then selects three non-collinear points, $v_{1}, v_{2}$, and $v_{3}$. 
The fourth point, $v_{4}$, is selected such that it is noncollinear with two of the three other points. Then, the parameters of four circles, $C_{i j k}$, obtained by each of the three points, $\left(x-a_{i j k}\right)^{2}+\left(y-b_{i j k}\right)^{2}=r_{i j k}$, are computed as follows:

$$
\begin{gathered}
a_{i j k}=\frac{\left|\begin{array}{ll}
x_{j}^{2}+y_{j}^{2}-\left(x_{i}^{2}+y_{i}^{2}\right) & 2\left(y_{j}-y_{i}\right) \\
x_{k}^{2}+y_{k}^{2}-\left(x_{i}^{2}+y_{i}^{2}\right) & 2\left(y_{k}-y_{i}\right)
\end{array}\right|}{4\left(\left(x_{j}-x_{i}\right)\left(y_{k}-y_{i}\right)-\left(x_{k}-x_{i}\right)\left(y_{j}-y_{i}\right)\right)}, \text { (8) } \\
b_{i j k}=\frac{\left|\begin{array}{ll}
2\left(x_{j}-x_{i}\right) & x_{j}^{2}+y_{j}^{2}-\left(x_{i}^{2}+y_{i}^{2}\right) \\
2\left(x_{k}-x_{i}\right) & x_{k}^{2}+y_{k}^{2}-\left(x_{i}^{2}+y_{i}^{2}\right)
\end{array}\right|}{4\left(\left(x_{j}-x_{i}\right)\left(y_{k}-y_{i}\right)-\left(x_{k}-x_{i}\right)\left(y_{j}-y_{i}\right)\right)}, \text { (9) } \\
r_{i j k}=\sqrt{\left(x_{l}-a_{i j k}\right)^{2}+\left(y_{l}-b_{i j k}\right)^{2}}, \text { for any } l=1,2,3,4 .
\end{gathered}
$$

Once the parameters of the four circles, $C_{123}, C_{124}$, $C_{134}$, and $C_{234}$, are found, the distance between point $v_{i}, i=1,2,3,4$ to the circle obtained from the three other points is computed: $d_{1} \rightarrow C_{234}, d_{2} \rightarrow C_{134}$, $d_{3} \rightarrow C_{124}$, and $d_{4} \rightarrow C_{123}$. If one of these distances is less than a threshold, $T_{d}$, the four points are called cocircular [Chen and Chung, 2001] and the three pixels composing the circle are referred to as agent pixels. The distance between each pair of agent points must be greater than a threshold, $T_{a}$.

If all these conditions are satisfied, the number of points, $n_{p}$ that are lying on the circle obtained by agent pixels are found. If $n_{p}$ is larger than a global threshold $T_{g}$, the circle is considered as a true circle and all the points that are lying on this circle are removed from set $V$. The algorithm iterates until only a small number of points remain in set $V$.

Since this approach requires that several parameters be set, it was modified by removing some thresholds and some steps are also changed so the algorithm adapts to our application. Eliminating several thresholds of the original RCD method decreases the number of failures and makes the algorithm converge faster while maintaining the same level of accuracy. In the following, the modified RCD approach is explained:

1. Suppose $V$ to be a set of input points (pixels), which is a set of projected 3D points on the plane. First, the counters and thresholds are initialized as follows:

$f$ : failure counter is set to 0 .

$T_{f}$ : failure threshold, maximum number of failures that are tolerated by the algorithm.

$T_{\text {min }}$ : the minimum number of points that can remain in set $V$ in order to stop the algorithm.

$T_{p}$ : the distance threshold between each point and the fitted circle.
2. If $f=T_{f}$ or $|V|<T_{\text {min }}$, the algorithm stops; otherwise four pixels are selected from set $V$ so they are co-circular.

3. The four possible circles associated with these four points are computed and then the number of points lying on the circles are identified. To achieve this, the distance between each point in set $V$ and each circle is determined. The number of points, $N_{p}$, whose distance is less than threshold $T_{p}$ is stored.

4. The circle with the largest $N_{p}$ is selected as the best fitted circle. If $N_{p}$ is less than one percent of $V$, the failure counter $f$ is incremented by one and the algorithm returns to step 1 . If $N_{p}$ is greater than $45 \%$ of $V$, the sum of distances from the best fitted circle is computed and divided by $N_{p}$. If this value is less than $\varepsilon_{p}$, the circle is selected as the true circle, the circle's parameters are stored and all points in $V$ lying on the circle are removed and one returns to step 1 .

With the above procedure the great and small radii of the torus, $R$ and $r$, and the axis direction, $\vec{d}$ can be estimated. The other parameter that remains to be extracted is the center of the torus. Using either RCD or Taubin's method, we have the centers of the circles on the plane, $c_{x}$ and $c_{y}$, while we need to extract the center of the torus in 3D. As mentioned before, all points of the torus were projected onto a plane whose normal is $\vec{d}$. This vector is one of the eigenvectors of the PCA, so the two other eigenvectors, $\vec{A}$ and $\vec{B}$, are the vectors lying on the plane onto which the torus points are projected. If we consider $(x, y, z)$ as the coordinates of torus points, $\vec{d}=\left(d_{1}, d_{2}, d_{3}\right)$, $\vec{A}=\left(a_{1}, a_{2}, a_{3}\right)$, and $\vec{B}=\left(b_{1}, b_{2}, b_{3}\right)$ as the PCA eigenvectors, the projection of points on these vectors are expressed as follows:

$$
\begin{gathered}
\left(\begin{array}{lll}
a_{1} & a_{2} & a_{3} \\
b_{1} & b_{2} & b_{3} \\
d_{1} & d_{2} & d_{3}
\end{array}\right)\left(\begin{array}{l}
x \\
y \\
z
\end{array}\right)=\left(\begin{array}{l}
a_{1} x+a_{2} y+a_{3} z \\
b_{1} x+b_{2} y+b_{3} z \\
d_{1} x+d_{2} y+d_{3} z
\end{array}\right) \\
\text { so, considering } M=\left(\begin{array}{lll}
a_{1} & a_{2} & a_{3} \\
b_{1} & b_{2} & b_{3} \\
d_{1} & d_{2} & d_{3}
\end{array}\right) \text { we have, } \\
\left(\begin{array}{l}
x \\
y \\
z
\end{array}\right)=M^{-1}\left(\begin{array}{l}
a_{1} x+a_{2} y+a_{3} z \\
b_{1} x+b_{2} y+b_{3} z \\
d_{1} x+d_{2} y+d_{3} z
\end{array}\right)=M^{-1}\left(\begin{array}{l}
x^{\prime} \\
y^{\prime} \\
z^{\prime}
\end{array}\right) .
\end{gathered}
$$

$\left(x^{\prime}, y^{\prime}, z^{\prime}\right)$ are the coordinates of the projection of $(x, y, z)$ on $\vec{d}, \vec{A}$, and $\vec{B}$, respectively. All values of matrix $M$ are know. The x-coordinate and y-coordinate of the circles center, $c_{x}$ and $c_{y}$ respectively, are the 


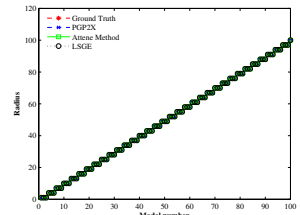

(a)

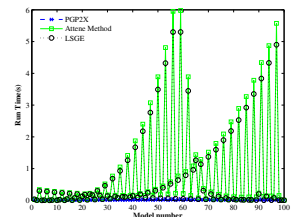

(c)

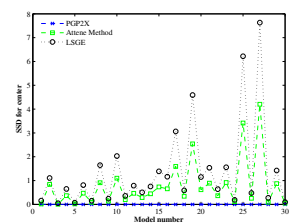

(e)

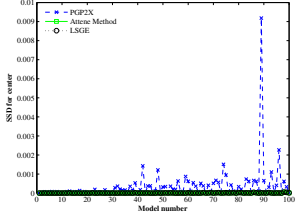

(b)

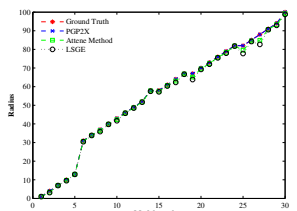

(d)

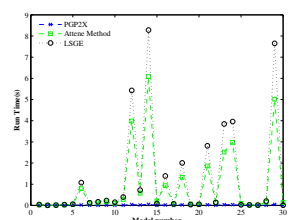

(f)
Figure 9: Comparison of sphere parameters between ground truth values, our method (PGP2X), Attene's method and LSGE. The first row shows the result for complete spheres and the second row presents the results for partial spheres. Plots (a) and (d) compare radius values, the comparison of the sum of square differences for the center values is presented in plots (b) and (e). plots (c) and (f) show the run times for the different approaches.

same as the $\mathrm{x}$ and $\mathrm{y}$ coordinates of the torus center, so, $x^{\prime}=c_{x}$ and $y^{\prime}=c_{y}$. The only missing parameter is $z^{\prime}$ which is exactly the mean value of the torus points projected on $\vec{d}$. Now, using equation 12 the 3D coordinate of the torus center, $(x, y, z)$ can be computed.

\section{EXPERIMENTAL RESULTS}

In order to study the result of our method for different models, we prepared $620 \mathrm{CAD}$ models with the 3DsMax software: 100 planes, 100 cones, 100 cylinders, 100 spheres, 100 tori, 30 partial cones, 30 partial cylinders, 30 partial spheres and, 30 partial tori which is called GPrimDB (Geometric Primitive Data Base). We selected the parameters of the models in order to design small, large, sparse, and dense models (from $1 \mathrm{~mm}$ to $100 \mathrm{~mm}$ ). The database includes cones with the cap (100 full cones and 30 partial cones) which is not used in this paper. In this section, we compare our parameter detection method with two other approaches: Attene's method [Attene and Patanè, 2010] and LSGE (Least Squares Geometric Element Software) [LSGE, 2004]. Attene's method has been applied for Planes, cones, cylinders, and spheres but it does not achieve good results for tori. The LSGE uses least-squares fitting in order to find the parameters of the primitives. For all primitives, an initial value for each parameter is required which limits the use of the algorithm. Consequently, we use Attene's method results as the initial values for LSGE for spheres and cylinders. Since Attene's method does not achieve good results for tori, initial values are not available for torus fitting in LSGE. For cones, Attene's method does not return the radius of the base which is one of the initial values that is required by LSGE.

Since Attene's method and LSGE perform well for spheres and cylinders, we compare our results to these two approaches and to ground truth values. The parameters that are introduced in section 3.5 are chosen empirically and are set to the following values: $\varepsilon$ : the distance associated with the second peak in the difference of sorted distances' diagram, $T_{f}: 30000$, $T_{\text {min }}:|V| \times 0.1, T_{p}: 0.5$, and $\varepsilon_{p}: 0.3$.

Figure 9 presents six diagrams for sphere models. The first row shows the results of the comparison for 100 complete spheres of the database with respect to both the radius and the center of the sphere with the run time of the algorithms. The second row presents the results for 30 partial spheres of the database. In order to compare the computed center, $\left(c_{1}^{\prime}, c_{2}^{\prime}, c_{3}^{\prime}\right)$, with the ground truth center, $\left(c_{1}, c_{2}, c_{3}\right)$, the Sum of Square Differences (SSD) is calculated as follows:

$$
S S D=\sqrt{\left(c_{1}-c_{1}^{\prime}\right)^{2}+\left(c_{2}-c_{2}^{\prime}\right)^{2}+\left(c_{3}-c_{3}^{\prime}\right)^{2}} .
$$

Where the SSD is close to zero, the method is judged as accurate. The SSD values are presented in the second plot for both complete and partial spheres. The first plots show the computed radii in comparison with ground truth radii. The run time of the different approaches is plotted in the last plots. The results show that all methods perform well for estimating the radius. For the center, all methods provide good results. PGP2X for complete spheres varies somewhat for some models but is significantly more efficient than other methods in processing time.

For cylinders, Attene's method and LSGE return the radius of the cylinder, the direction of the axis and a point on the axis while PGP $2 X$ returns the radius, the direction axis, the height, and the center of the base. If the direction axis is not correct, the radius of the cylinder will not be correct either. In the following, we compare the parameters that are common to the other approaches and ours. Figure 10, shows the computed radius and run time for the different approaches for both complete and partial cylinders. 


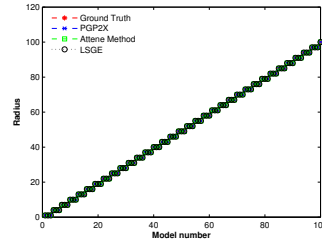

(a)

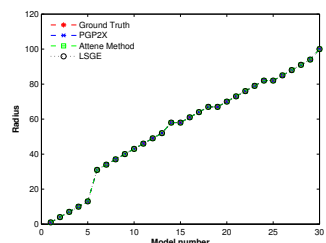

(c)

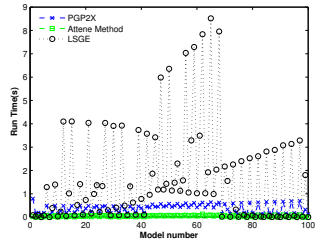

(b)

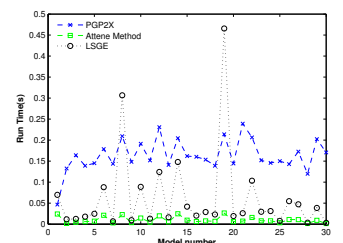

(d)
Figure 10: Comparison of cylinder parameters between ground truth values, our method (PGP2X), Attene's method and LSGE. The first row shows the result for complete cylinders and the second row presents the results for partial cylinders. Plots (a) and (c) present the comparison for the radius and plots (b) and (d) show the run times for the different approaches.

The results show that all methods perform well for the radius. PGP2X is slightly slower than the others since it estimates more parameters (i.e. the exact location of the center on the base). We also compare PGP2X with ground truth values for the height and center of cylinders. Figure 11 shows the difference between the computed height and center estimated by PGP2X and the ground truth values for both complete and partial models.

For cone models, Attene's method [Attene and Patanè, 2010], identifies the direction of the axis, the apex and the semi-apical angle which expresses the deviation of the cone border from the direction of the axis. The ground truth values of the cones designed with 3DsMax do not correspond to these parameters. The rotation of the 3D model is entered as an input to 3DsMax, so the ground truth axis direction can be estimated based on the rotation information given by 3DsMax (multiplying the rotation matrix by unit axis direction $(0,0,1))$. The semi-apical axis is the arctangent of the cone radius over its height. The position of the apex cannot be estimated from ground truth parameters.

For cones, our approach estimates the center, the radius of the cone base, the height, and the direction of the axis plus the semi-apical angle and the position of the apex. So, in order to make the comparison of the axis direction computed by different methods, the SSD is calculated using the ground truth values (see part (a) in figures 12 and 13 for complete and par-

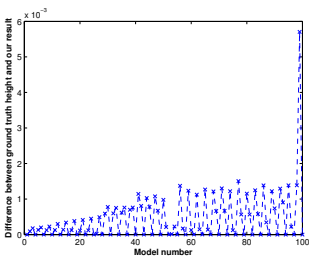

(a)

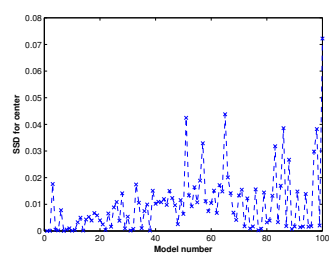

(c)

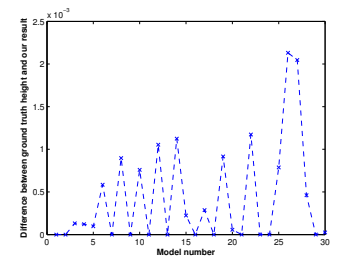

(b)

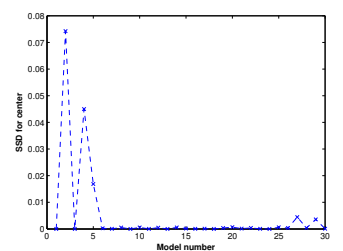

(d)
Figure 11: The difference between ground truth values for height and center of cylinders and results obtained by our method (PGP2X). Plots (a) and (c) shows the results of the complete models (height and center). Plots (b) and (d) show the results of partial models for height and center, respectively.

tial models, respectively). For the semi-apical angle, Attene's method and our approach are compared to ground truth values in part (b) of figures 12 and 13 for complete and partial models, respectively. For the position of the apex, we have Attene's results and our result without the ground truth values as a reference, so, we have not provided any comparison for this parameter. For the center of the cone base, the height and the radius, we compare the results of our method with the ground truth for both complete and partial models in figures 12 and 13 in part(c), (d), and (e), respectively. The run time is presented in part (f) in figures 12 and 13 for both complete and partial models. The results in figure 12 and 13 show that our method can estimate more parameters in comparison with Attene's method and thus provides a more detailed description of the primitive. Our results are very close to ground truth values. The run time of our method is less than Attene's approach even though our approach provides more parameters.

For torus primitives, since no other method deals with this primitive, our method is compared to ground truth values only. Therefore, we present the comparison between our method and ground truth in a tabular manner as the average of the sum of square differences. Torus models are described by the center, the great and small radii, and the direction of the axis. For the center and the direction of the axis, we compute the average of SSD values for all models and for the two radii we compute the average of Euclidean distances between ground truth values and our results. 


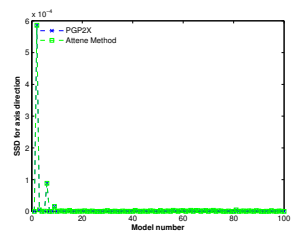

(a)

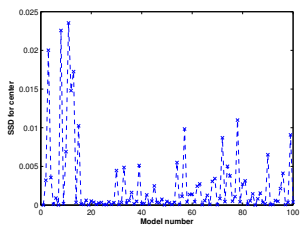

(c)

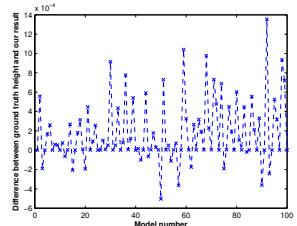

(e)

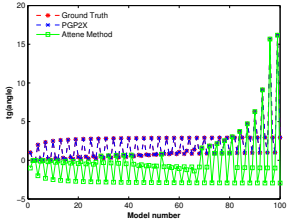

(b)

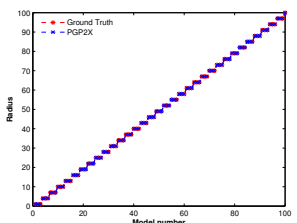

(d)

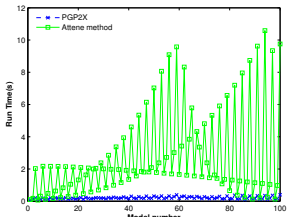

(f)
Figure 12: Cone parameters comparison between ground truth values, our method (PGP2X) and Attene's method. Plot (a) shows the sum of square differences for the axis direction between PGP2X, Attene's method and ground truth. Plot (b) presents the tangent of the angle for PGP2X, Attene's method in comparison with ground truth values. The comparison for the center, the radius and the height between PGP2X and ground truth is provided in plots (c)-(e), respectively. Finally plot (f) shows the run time for PGP2X in comparison with Attene's approach.

These results are presented in table 1 . The results show that our method can identify all parameters of the torus models with an acceptable error.

In order to study our method in the case of real models, we scanned objects such as a ball (sphere), a life saver (torus), a pipe (cylinder), and a paper cone. It is clear that a ball is not a perfect sphere, a life saver is also not a perfect model since it is slightly

\begin{tabular}{c|c|c} 
& Complete Torus & Partial Torus \\
\hline Great Radius & 0.0174 & 0.2226 \\
\hline Small Radius & 0.0189 & 0.0844 \\
\hline Center & $1.426 \times 10^{-4}$ & 0.2268 \\
\hline Axis Direction & $5.933 \times 10^{-7}$ & $1.187 \times 10^{-5}$
\end{tabular}

Table 1: Partial and complete torus models parameters error. The values that are provided in the tables are the average of differences between ground truth values and our results. The difference that is computed for the center and the axis direction is the sum of square distances. The Euclidean distance is computed for the two radii.

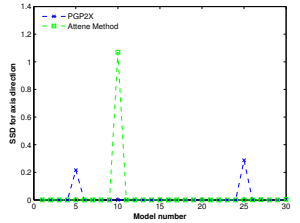

(a)

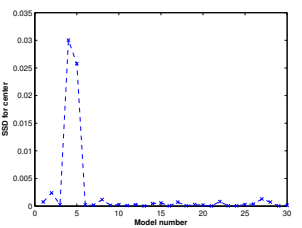

(c)

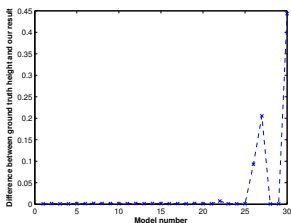

(e)

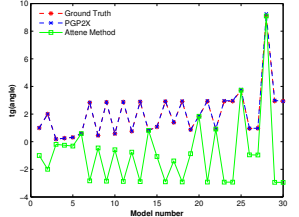

(b)

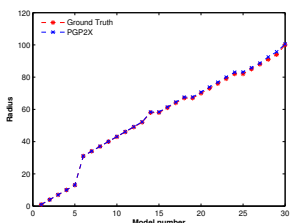

(d)

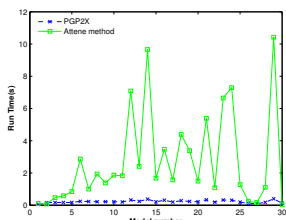

(f)
Figure 13: Comparison of partial Cone parameters between ground truth values, our method (PGP2X), and Attene's method. Plot (a) shows the sum of square differences for the axis direction between PGP2X, Attene's method and ground truth values. Plot (b) presents the tangent of the angle for PGP2X, Attene's method in comparison with ground truth values. The comparison for the center, the radius and the height between our result and ground truth values is provided in plots (c)-(e), respectively. Plot (f) shows the run times for PGP2X in comparison with Attene's approach.

twisted, see figure 14. Since the exact parameters of these models are not known and there is no easy means of measuring them in order to verify the accuracy of our method, we scanned the objects with the Creaform Go!Scan 3D handheld scanner, applied our method to the primitives to identify their parameters and used the Polyworks inspection software in order to measure the parameters manually.

For the real sphere, cone, and cylinder, we selected points on the models and used Polyworks's primitive fitting function to determine the parameters. Since the software does not provide torus fitting, for the scanned torus, we mapped two spheres on the model in order to estimate the radii. One small sphere inside the torus and one great sphere around the torus. In absence of ground truth parameters, a visual comparison is also provided in figure 14 to assess which method determines the best parameters for the primitives. The numerical values are also provided in the same figure. The results indicate that our method provides parameter values comparable to Polyworks. Our approach functions automati- 
cally while Polyworks involves a manual process.

\section{CONCLUSION}

In this paper, we presented a novel method for computing the parameters of geometric primitives such as plane, sphere, cylinder, cone, and torus. Most methods presented in the literature do not provide accurate results for tori. We compared our method (PGP2X) with two other approaches in the case of common primitives (spheres, cylinders and, cones). For tori, we provided a comparison with ground truth values. Our method works well for both complete and partial primitives. The experiments show that our method is accurate and performs well even in the case of real noisy models acquired with $3 \mathrm{D}$ sensors.

\section{ACKNOWLEDGEMENTS}

This work was supported by the NSERC-Creaform Industrial Research Chair on 3D Scanning. Z. Toony was supported by a FRQNT post-graduate scholarship. The authors thank Trung-Thien Tran for providing us with the implementation of Attene's method for cylinders and spheres [Attene and Patanè, 2010] and Annette Schwerdtfeger for proofreading this manuscript.

\section{REFERENCES}

Attene, M., Falcidieno, B., and Spagnuolo, M. (2006). Hierarchical mesh segmentation based on fitting primitives. The Visual Computer, 22(3):181-193.

Attene, M. and Patanè, G. (2010). Hierarchical structure recovery of point-sampled surfaces. In Computer Graphics Forum, volume 29, pages 19051920. Wiley Online Library.

Benko, P., Kós, G., Várady, T., Andor, L., and Martin, R. (2002). Constrained fitting in reverse engineering. Computer Aided Geometric Design, 19(3):173-205.

Bolles, R. C. and Fischler, M. A. (1981). A RANSAC-based approach to model fitting and its application to finding cylinders in range data. In 7th Int. Joint Conf. on Artificial Intelligence (IJCAI'81), volume 1981, pages 637-643.

Borrmann, D., Elseberg, J., Lingemann, K., and Nüchter, A. (2011). The 3D Hough transform for plane detection in point clouds: A review and a new accumulator design. 3D Research, 2(2):113.

Chaperon, T., Goulette, F., and Laurgeau, C. (2001). Extracting cylinders in full 3D data using a random sampling method and the gaussian image. In Proc. of the Vision Modelling and Visualization Conf., volume 1, pages 35-42. Citeseer.

Chen, T.-C. and Chung, K.-L. (2001). An efficient randomized algorithm for detecting circles. Computer Vision and Image Understanding, 83(2):172-191.

Chernov, N. (2009). Circle Fitting by Taubin method. http://www. mathworks. $\mathrm{com} / \mathrm{mat}$ labcentral/fileexchange/ 22678-circle-fit--taubin-method-. [Online; accessed January-2009].

Cohen-Steiner, D., Alliez, P., and Desbrun, M. (2004). Variational shape approximation. In ACM Trans. on Graphics (TOG), volume 23, pages 905-914.

Fayolle, P.-A. and Pasko, A. (2013). Segmentation of discrete point clouds using an extensible set of templates. The Visual Computer, 29(5):449465.

Fischler, M. A. and Bolles, R. C. (1981). Random sample consensus: a paradigm for model fitting with applications to image analysis and automated cartography. Communications of the ACM, 24(6):381-395.

Garland, M., Willmott, A., and Heckbert, P. S. (2001). Hierarchical face clustering on polygonal surfaces. In Proc. of the 2001 Sym. on Interactive $3 D$ graphics, pages 49-58. ACM.

Johnston, J. D. (1988). Transform coding of audio signals using perceptual noise criteria. IEEE Journal on Selected Areas in Communications, 6(2):314-323.

Kasa, I. (1976). A circle fitting procedure and its error analysis. IEEE Trans. on Instrumentation and Measurement, 1001(1):8-14.

Kazhdan, M., Funkhouser, T., and Rusinkiewicz, S. (2003). Rotation invariant spherical harmonic representation of 3D shape descriptors. In Proc. of the 2003 Eurographics/ACM SIGGRAPH Sym. on Geometry processing, pages 156-164. Eurographics Association.

Kotthäuser, T. and Mertsching, B. (2012). Triangulation-based plane extraction for 3D point clouds. In Intelligent Robotics and Applications, pages 217-228. Springer.

Li, Y., Wu, X., Chrysathou, Y., Sharf, A., Cohen-Or, D., and Mitra, N. J. (2011). Globfit: Consistently 


\begin{tabular}{|c|c|c|}
\hline Scanned model & PGP2X result & The result obtained with Polyworks \\
\hline & $\begin{array}{l}\text { Center }=(-1.128,-3.22,552.079) \\
\text { Radius }=192.5097\end{array}$ & $\begin{array}{l}\text { Center }=(-0.843,-2.978,552.789) \\
\text { Radius }=192.219\end{array}$ \\
\hline & $\begin{array}{l}\text { Orientation }=(-0.0585,0.701,0.71) \\
\text { Height }=81.482, \text { Radius }=45.217 \\
\text { Apex }=(6.398,-60.937,341.699) \\
\text { Semi }- \text { apicalangle }=29.028\end{array}$ & $\begin{array}{l}\text { Orientation }=(-0.091,0.708,0.701) \\
\text { Height }=79.14 \\
\text { Apex }=(8.271,-61.816,341.712) \\
\text { Semi }- \text { apicalangle }=27.603\end{array}$ \\
\hline & $\begin{array}{l}\text { Orientation }=(0,0,1) \\
\text { Height }=100, \text { Radius }=50.008 \\
\text { Center }=(-36.159,72.951,0)\end{array}$ & $\begin{array}{l}\text { Orientation }=(0,0,1) \\
\text { Height }=100.004, \text { Radius }=50.002 \\
\text { Center }=(-36.154,72.913,-0.001)\end{array}$ \\
\hline & $\begin{array}{l}\text { Center }=(-113.781,-119.821,487.169) \\
\text { Great radius }=239.0103 \\
\text { Small radius }=109.309\end{array}$ & $\begin{array}{l}\text { Center }=(-115.658,125.371,500.384) \\
\text { Great radius }=238.857 \\
\text { Small radius }=109.125\end{array}$ \\
\hline
\end{tabular}

Figure 14: Parameter Extraction for real scanned models. The parameters are extracted using our method (PGP2X) and the Polyworks software. Each row shows the result for one model using both methods.

fitting primitives by discovering global relations. In ACM Transactions on Graphics, volume 30, page 52.

Liu, Y.-J., Zhang, J.-B., Hou, J.-C., Ren, J.-C., and Tang, W.-Q. (2013). Cylinder detection in large-scale point cloud of pipeline plant. IEEE Trans. on Visualization and Computer Graphics, 19(10):1700-1707.

Lozano-Perez, T., Grimson, W., and White, S. (1987). Finding cylinders in range data. In Proc. of IEEE Intl. Conf. on Robotics and Automation, volume 4, pages 202-207.

LSGE (2004). LSGE: The Least Squares Geometric Elements Library. http: //www. eurometros.org/metros/key_ functions/fitting_routines/.

Lukács, G., Martin, R., and Marshall, D. (1998). Faithful least-squares fitting of spheres, cylinders, cones and tori for reliable segmentation. In 5th European Conf. on Computer Vision (ECCV'98), pages 671-686. Springer.

Olson, C. F. (2001). Locating geometric primitives by pruning the parameter space. Pattern Recognition, 34(6):1247-1256.

Osada, R., Funkhouser, T., Chazelle, B., and Dobkin, D. (2002). Shape distributions. ACM Transactions on Graphics (TOG), 21(4):807-832.

Pratt, V. (1987). Direct least-squares fitting of algebraic surfaces. ACM SIGGRAPH Computer Graphics, 21(4):145-152.

Rabbani, T. and Van Den Heuvel, F. (2005). Efficient Hough transform for automatic detection of cylinders in point clouds. Proc. of ISPRS WS on Laser Scanning, 3:60-65.

Scales, L. (1985). Introduction to non-linear optimization. Springer-Verlag New York, Inc.

Schmitt, S. R. (2005). Center and Radius of a Sphere from Four Points. http: //www. abecedarical.com/zenosamples/ zs_sphere4pts.html.

Schnabel, R., Wahl, R., and Klein, R. (2007). Efficient RANSAC for point-cloud shape detection. In Computer graphics forum, volume 26, pages 214-226. Wiley Online Library.

Taubin, G. (1991). Estimation of planar curves, surfaces, and nonplanar space curves defined by implicit equations with applications to edge and range image segmentation. IEEE Trans. on Pattern Analysis and Machine Intelligence, 13(11):1115-1138.

Toony, Z., Laurendeau, D., and Gagné, C. (2014). Recognizing 3D principal primitives using gaussian sphere and gaussian accumulator. Internal Report. Computer Vision and System Laboratory, Laval University, August 2014.

Zhang, J., Cao, J., Liu, X., Wang, J., Liu, J., and Shi, X. (2013). Point cloud normal estimation via low-rank subspace clustering. Computers \& Graphics, 37(6):697-706.

Zhu, K., Wong, Y. S., Loh, H. T., and Lu, W. F. (2012). 3D CAD model retrieval with perturbed laplacian spectra. Computers in Industry, 63(1):1-11. 